\title{
A constructive proof of Simpson's Rule
}

\author{
THIERRY COQUAND \\ BAS SPITTERS
}

\begin{abstract}
For most purposes, one can replace the use of Rolle's theorem and the mean value theorem, which are not constructively valid, by the law of bounded change [3]. The proof of two basic results in numerical analysis, the error term for Lagrange interpolation and Simpson's rule, however seem to require the full strength of the classical Rolle's Theorem. The goal of this note is to justify these two results constructively, using ideas going back to Ampère [1] and Genocchi [7].
\end{abstract}

2010 Mathematics Subject Classification 03F60; 65D30 (primary)

Keywords: Divided difference; Hermite-Genocchi; Simpson rule

\section{Introduction}

Rolle's Theorem states that if $f$ is differentiable on a real interval $[a, b]$ with $a<b$ and $f(a)=f(b)=0$ then there exists $c$ in $] a, b$ [ such that $f^{\prime}(c)=0$. It implies directly the mean value theorem that if $f$ is differentiable on a real interval $[a, b]$ with $a<b$, then there exists $c$ in $] a, b$ [ such that $f(b)-f(a)=(b-a) f^{\prime}(c)$. This is a key result in most text books in Analysis. It is rather direct to see that it does not hold constructively [2], and is replaced in this context by an approximated form: if $f$ is differentiable on a real interval $[a, b]$ with $a<b$ then for any $\epsilon>0$ there exists $c$ in $] a, b$ [ such that $\left|f(b)-f(a)-(b-a) f^{\prime}(c)\right|<\epsilon$. This more complex formulation can be thought to be a problem of constructive mathematics.

It can be argued however [3] that most applications of the mean value theorem can be replaced by the law of bounded change that we have $|f(b)-f(a)| \leqslant M(b-a)$ if $f$ is uniformly derivable on $[a, b]$ and $\left|f^{\prime}(x)\right| \leqslant M$ for all $x$ in $[a, b]$. The law of bounded change is constructively valid and a presentation based on the law of bounded change [3] appears as elegant as the classical treatment of basic analysis results. Interestingly, a criticism of the mean value theorem, which could have been written by a constructive mathematician, appears in Dieudonné Foundations of Modern Analysis [5]: "the trouble with that classical formulation is that ... it completely conceals the fact that nothing 
is known on the number $c$, except that it lies between $a$ and $b$, and for most purposes, all one needs to know is that $f^{\prime}(c)$ is a number which lies between that g.l.b. and l.u.b. of $f^{\prime}$ in the interval $[a, b]$ (and not the fact that it actually is a value of $f^{\prime}$ )." The goal of this note is to analyse two results that at first seem to require the full strength of the classical mean value theorem. The second of these results is Simpson's rule for approximating an integral, which is indeed proved in the exercises of [5] using Rolle's Theorem. We show, using some ideas going back to Ampère [1] and Genocchi [7], how to justify these results constructively.

This note is organised as follows. We first present the two results we want to analyse: Lagrange error formula and Simpson's rule. We then explain Genocchi's formula, in a way which stresses the connection with the work of Bridger and Stolzenberg [3], and show how it can be used instead of Rolle's Theorem.

\section{Lagrange error formula and Simpson's rule}

In this section, we present two basic results, Theorems 2.2 and 2.3, and explain how they can be classically derived using the following generalization of Rolle's Theorem.

Theorem 2.1 (Classical generalized Rolle's theorem) Let $f$ be $n$ times differentiable and have $n+1$ zeroes in an interval $[a, b]$. Then $f^{(n)}$ has a zero in $[a, b]$.

Proof If $f$ has $n+1$ zeroes $x_{0}<x_{1}<\cdots<x_{n}$ then using Rolle's Theorem $f^{\prime}$ will have $n$ zeroes $y_{0}, \ldots, y_{n-1}$ with $y_{i}$ in $] x_{i}, x_{i+1}$ [. Hence we obtain the result by induction on $n$.

We now present the Lagrange polynomial as it can be found in numerical analysis textbooks; e.g. [6].

Theorem 2.2 (Lagrange error formula) Let $f$ be $n$ times differentable on an interval $[a, b], P$ the polynomial of degree $n-1$ which agrees with $f$ on $n$ values $x_{0}<\cdots<$ $x_{n-1}$ and $M$ such that $\left|f^{(n)}(x)\right| \leqslant M$ for all $x$ in $[a, b]$. Then for all $x$ in $[a, b]$

$$
|f(x)-P(x)| \leqslant \frac{\left|\prod\left(x-x_{k}\right)\right|}{n !} M
$$


Proof This is proved using Rolle's Theorem in the following way [5, 6]. First, classically, we can assume that $x$ is not equal to one of the $x_{i}$ since the inequality is clear if $x=x_{i}$. We then consider the function

$$
g(y)=\frac{\prod\left(x-x_{k}\right)}{n !}(f(y)-P(y))-\frac{\prod\left(y-x_{k}\right)}{n !}(f(x)-P(x))
$$

This function is $n$ times differentiable and has $n+1$ zeroes $x, x_{0}, \ldots, x_{n-1}$. Using Theorem 2.1, there exists $c$ such that $g^{(n)}(c)=0$ which can be written as

$$
f(x)-P(x)=\frac{\prod\left(x-x_{k}\right)}{n !} f^{(n)}(c) .
$$

This finishes the proof.

By a similar use of Theorem 2.1, one can derive the following classical result $[5,6]$.

Theorem 2.3 (Simpson's rule [6]) If $f$ is 4-differentiable on an interval $[a, b]$ and $\left|f^{(4)}(x)\right| \leqslant M$ for all $x$ in $[a, b]$, then we have

$$
\left|\int_{a}^{b} f(x) d x-\frac{b-a}{6}\left[f(a)+4 f\left(\frac{a+b}{2}\right)+f(b)\right]\right| \leqslant \frac{(b-a)^{5}}{2880} M .
$$

Rolle's theorem is constructively provable [2] provided $f^{\prime}$ is locally non-constant: In every interval there are $x, y$ such that $f^{\prime}(x)<f^{\prime}(y)$. In this case, $f^{\prime}$ is in particular locally nonzero, i.e. in every interval there is an $x$ such that $f^{\prime}(x)>0$ or $f^{\prime}(x)<0$. It follows that $f$ is locally non-constant, as is readily seen by integration. We see that if $f^{(n)}$ is locally nonzero, then for all $k<n, f^{(k)}$ is locally nonconstant. We obtain an equal conclusion version of the generalized Rolle's theorem: Let $f$ be $n$ times differentiable and have $n+1$ zeroes in an interval $[a, b]$. If, moreover, $f^{(n)}$ is locally nonzero, then $f^{(n)}$ has a zero in $[a, b]$.

From this equal conclusion version, we can obtain an equal hypothesis version of Rolle's theorem.

Proposition 2.4 Let $f$ be $n$ times differentiable and have $n+1$ zeroes, $x_{i}$, in an interval $[a, b]$. Then there exists $x \in[a, b]$ such that $\left|f^{(n)}(x)\right| \leq \varepsilon$.

Proof Either $\inf _{x \in[a, b]}\left|f^{(n)}(x)\right|<\varepsilon \operatorname{or~inf}_{x \in[a, b]}\left|f^{(n)}(x)\right|>\varepsilon / 2$. In the former case, we are done. In the latter case, $f^{(n)}$ is locally non-zero, hence by the remark above, we can follow the proof of Theorem 2.1 to conclude that $f^{(n)}$ has a zero. A contradiction.

In this way we can derive the Lagrange error formula 2.2. One can argue however that this derivation is more complex than the classical result. In Section 4.1 we give smooth proofs of Theorems 2.2 and 2.3, which hold both classically and constructively, and do not rely on Theorem 2.1. 


\section{Hermite-Genocchi formula}

The definition in [3] of uniform differentiability of a function $f$ on an interval $I=[a, b]$ can be formulated as follows: there exists an uniformly continuous function $F: I^{2} \rightarrow \mathbb{R}$ such that $f(y)-f(x)=(y-x) F(x, y)$ for all $x, y$ in $I$. We can then define $f^{\prime}(x)$ to be $F(x, x)$. The following result is a generalization of this characterisation to $n$ differentiability. The proof is a simple application of the fundamental theorem of the calculus [3].

Theorem 3.1 A function $f: I \rightarrow \mathbb{R}$ is uniformly $n$-differentiable if, and only if, there exist $n+1$ uniformly continuous functions $f_{0}(x), f_{1}\left(x_{0}, x_{1}\right), \ldots, f_{n}\left(x_{0}, \ldots, x_{n}\right)$ defined respectively on $I, I^{2}, \ldots, I^{n+1}$ and such that

$$
\begin{array}{r}
f_{0}(x)=f(x), f_{0}\left(x_{1}\right)-f_{0}\left(x_{0}\right)=\left(x_{1}-x_{0}\right) f_{1}\left(x_{0}, x_{1}\right), \ldots, \\
f_{n-1}\left(x_{0}, \ldots, x_{n-2}, x_{n}\right)-f_{n-1}\left(x_{0}, \ldots, x_{n-2}, x_{n-1}\right)=\left(x_{n}-x_{n-1}\right) f_{n}\left(x_{0}, \ldots, x_{n}\right)
\end{array}
$$

for all $x_{0}, \ldots, x_{n}$ in $I$.

We then have

$$
f^{(n)}(x)=n ! f_{n}(x, \ldots, x)
$$

and, conversely, we can define

$$
f_{n}\left(x_{0}, \ldots, x_{n}\right)=\int_{\Sigma_{n}} f^{(n)}\left(t_{0} x_{0}+\cdots+t_{n} x_{n}\right) d t_{0} \ldots d t_{n}
$$

where $^{1} \Sigma_{n}=\left\{\left(t_{0}, \ldots, t_{n}\right) \in[0,1]^{n+1} \mid t_{0}+\cdots+t_{n}=1\right\}$.

Proof Assume that the divided differences are uniformly continuous on $I, I^{2}, \ldots, I^{n+1}$. Since

$$
f(y)-f(x)=(y-x) f_{1}(x, y)
$$

we get that $f$ is uniformly differentiable and $f^{\prime}(x)=f_{1}(x, x)$. We then have

$$
\begin{aligned}
f^{\prime}(y)-f^{\prime}(x) & =f_{1}(y, y)-f_{1}(x, x) \\
& =f_{1}(y, y)-f_{1}(y, x)+f_{1}(y, x)-f_{1}(x, x) \\
& =(y-x)\left(f_{2}(y, x, y)+f_{2}(x, x, y)\right)
\end{aligned}
$$

\footnotetext{
${ }^{1}$ This integral of a uniformly continuous function $g$ over $\Sigma_{n}$ can be defined by induction on $n$ in the following way: for $n=0$ the integral is $g(1)$, and for $n>0$ we define

$$
h\left(t_{0}, \ldots, t_{n-1}\right)=\int_{0}^{1} g\left(t_{0}, \ldots, t_{n-1}(1-u), t_{n-1} u\right) d u
$$

and the integral of $g$ over $\Sigma_{n}$ is the integral of $h$ over $\Sigma_{n-1}$.
} 
and so $f$ is uniformly 2-differentiable and $f^{(2)}(x)=2 f_{2}(x, x, x)$. Proceeding in this way we see that $f$ is uniformly $k$-differentiable and that we have $k ! f_{k}(y, \ldots, y)=f^{(k)}(y)$ for $k=0, \ldots, n$.

Conversely, assume that $f$ is uniformly $n$-differentiable. We define

$$
f_{k}\left(x_{0}, \ldots, x_{k}\right)=\int_{\Sigma_{k}} f^{(k)}\left(t_{0} x_{0}+\cdots+t_{k} x_{k}\right) d t_{0} \ldots d t_{k}
$$

for $k=0, \ldots, n$. These functions are uniformly continuous. Furthermore, we have $f_{0}(x)=f(x)$ and

$$
f_{0}(x)-f_{0}\left(x_{0}\right)=\left(x-x_{0}\right) f_{1}\left(x_{0}, x\right)
$$

since

$$
\left(x-x_{0}\right) f_{1}\left(x_{0}, x\right)=\int_{0}^{1}\left(f\left((1-t) x_{0}+t x\right)\right)^{\prime} d t
$$

holds for $x$ apart from $x_{0}$, by the fundamental theorem of the calculus [3] and hence for all $x, x_{0}$ by continuity. It follows that we have

$$
\begin{aligned}
& f_{k}\left(x_{0}, \ldots, x_{k-1}, x\right)-f_{k}\left(x_{0}, \ldots, x_{k-1}, x_{k}\right) \\
= & \int_{\Sigma_{k}}\left(f^{(k)}\left(t_{0} x_{0}+\cdots+t_{k} x\right)-f^{(k)}\left(t_{0} x_{0}+\cdots+t_{k} x_{k}\right)\right) d t_{0} \ldots d t_{k} \\
= & \left(x-x_{k}\right) \int_{\Sigma_{k}} \int_{0}^{1}\left(f^{(k+1)}\left(t_{0} x_{0}+\cdots+t_{k}(1-u) x_{k}+t_{k} u x\right) d u d t_{0} \ldots d t_{k}\right. \\
= & \left(x-x_{k}\right) \int_{\Sigma_{k+1}} f^{(k+1)}\left(t_{0} x_{0}+\cdots+t_{k} x_{k}+t_{k+1} x\right) d t_{0} \ldots d t_{k+1} \\
= & \left(x-x_{k}\right) f_{k+1}\left(x_{0}, \ldots, x_{k}, x\right)
\end{aligned}
$$

which shows that these functions satisfy the required equations.

Following Ampere [1] we observe from Formula 1:

$$
\begin{aligned}
f_{1}\left(x_{0}, x_{1}\right) & =\frac{f\left(x_{1}\right)}{x_{1}-x_{0}}+\frac{f\left(x_{0}\right)}{x_{0}-x_{1}} \\
f_{2}\left(x_{0}, x_{1}, x_{2}\right) & =\frac{f\left(x_{2}\right)}{\left(x_{2}-x_{0}\right)\left(x_{2}-x_{1}\right)}+\frac{f\left(x_{1}\right)}{\left(x_{1}-x_{0}\right)\left(x_{1}-x_{2}\right)}+\frac{f\left(x_{0}\right)}{\left(x_{0}-x_{1}\right)\left(x_{0}-x_{2}\right)} \\
f_{3}\left(x_{0}, x_{1}, x_{2}, x_{3}\right) & =\cdots
\end{aligned}
$$

when all he $x_{i}$ 's are distinct. Hence, the functions $f_{k}\left(x_{0}, \ldots, x_{k}\right)$ are symmetric - that is, they are invariant under permutation of the variables. Also by formula 1 ,

$$
f(x)=f_{0}\left(x_{0}\right)+\left(x-x_{0}\right) f_{1}\left(x_{0}, x_{1}\right)+\cdots+\left(x-x_{0}\right) \ldots\left(x-x_{n-1}\right) f_{n}\left(x_{0}, \ldots, x_{n-1}, x\right) .
$$


Formula 2 is known as Hermite-Genocchi formula. Genocchi found these formulae by analysing the notion of "fonctions interpolaires" due to Ampère [1] $]^{2}$.

A direct consequence of Theorem 3.1 is the following result.

Corollary 3.2 Given a function $f: I \rightarrow \mathbb{R}$ and $n+1$ distinct elements $x_{0}, \ldots, x_{n}$ in $I$, let $P\left(x_{0}, \ldots, x_{n}, x\right)$ be the interpolation polynomial $a_{n-1}\left(x_{0}, \ldots, x_{n}\right) x^{n-1}+\cdots+$ $a_{0}\left(x_{0}, \ldots, x_{n}\right)$ of $f$ at $x_{0}, \ldots, x_{n}$. Then $P\left(x_{0}, \ldots, x_{n}, x\right)$, seen as a function of the parameters $x_{0}, \ldots, x_{n}$, can be extended to an uniformly continuous function on $I^{n+1}$ (that is, each function $a_{i}\left(x_{0}, \ldots, x_{n}\right)$ can be extended to an uniformly continuous function on $I^{n+1}$ ) if, and only if, $f$ is uniformly $n$-differentiable.

\section{Applications}

We explain now how to derive Theorem 2.2 from Theorem 3.1. We assume that $f: I \rightarrow \mathbb{R}$ is uniformly $n$-differentiable. Given any $n$ elements $x_{0}, \ldots, x_{n-1}$ in $I$ we associate the Newton polynomial of degree $n-1$ :

$P(x):=f_{0}\left(x_{0}\right)+f_{1}\left(x_{0}, x_{1}\right)\left(x-x_{0}\right)+\cdots+f_{n-1}\left(x_{0}, \ldots, x_{n-1}\right)\left(x-x_{0}\right)\left(x-x_{1}\right) \cdots\left(x-x_{n-2}\right)$.

We have

$$
f(x)-P(x)=\left(x-x_{0}\right) \ldots\left(x-x_{n-1}\right) f_{n}\left(x_{0}, \ldots, x_{n-1}, x\right)
$$

On the other hand, we also have $P\left(x_{i}\right)=f\left(x_{i}\right)$ for $i=0, \ldots, n-1$.

If $\left|f^{(n)}(u)\right| \leqslant M$ for all $u$ in $I$, then by Theorem 3.1,

$$
\left|f_{n}\left(x_{0}, \ldots, x_{n-1}, x\right)\right| \leqslant M \int_{\Sigma_{n}} 1=\frac{M}{n !}
$$

The last equality follows from applying Formula 2 to the function $g(x):=\frac{x^{n}}{n !}$ and observing that $g^{(n)}=1$ and $g_{n}(x, \ldots, x)=\frac{1}{n !}$, by Formula 1. This proves Theorem 2.2.

Notice that the Newton polynomial $P(x)$ is defined for any $x_{0}, \ldots, x_{n-1}$ without requiring them to be distinct. We have $P^{(l-1)}\left(x_{i}\right)=f^{(l-1)}\left(x_{i}\right)$ if $x_{i}$ is duplicated $l$ times in this list.

Theorem 3.1 is also valid for $f: I \rightarrow E$, where $E$ is a Banach space. Theorem 2.2 is also valid for $f: I \rightarrow F$, where $F$ is a normed space. See for instance [5, 4] for differentation and integration with values in a Banach space.

\footnotetext{
${ }^{2}$ In the case where $f$ is a monic polynomial, the functions $f_{1}\left(x_{0}, x_{1}\right), \ldots, f_{n}\left(x_{0}, \ldots, x_{n-1}\right)$ are also polynomial and they form with $f$ a Gröbner basis of the universal decomposition algebra of the polynomial $f$; see e.g. [8].
} 


\subsection{A constructive proof of Simpson's rule}

As explained above, the typical proofs of Simpson's rule 2.3, see e.g. [6, 9], use Rolle's theorem, and so are not constructively valid. We adapt the proof in [9] which uses Rolle's theorem three times on the triple zero at 0 and the simple zero at 1 of the function $H$ below.

Define $F(t):=f\left(\frac{a+b}{2}+\frac{b-a}{2} t\right)$. This reduces the problem to showing that

$$
\left|\int_{-1}^{1} F(\tau) d \tau-\frac{1}{3}(F(-1)+4 F(0)+F(1))\right| \leqslant N / 90,
$$

where $N:=\left\|F^{(4)}\right\|$.

Define

$$
G(t)=\int_{-t}^{t} F(\tau) d \tau-\frac{t}{3}(F(-t)+4 F(0)+F(t))
$$

We need to prove that $90 G(1) \leqslant N$. To do so, define $H(t):=G(t)-t^{5} G(1)$. Then

$$
H(0)=H(1)=H^{\prime}(0)=H^{\prime \prime}(0)=0 .
$$

Hence, $H_{3}(0,0,0,1)=-\left(H_{2}(0,0,0)-H_{2}(0,0,1)\right)=0+\left(-H_{1}(0,0)+H_{1}(0,1)\right)=0$. (This line replaces three uses of Rolle's theorem.)

Moreover,

$$
H^{(3)}(t)=-\frac{t}{3}\left(F^{(3)}(t)-F^{(3)}(-t)\right)-60 t^{2} G(1)=-\frac{t}{3}\left(\int_{-t}^{t} F^{(4)}\right)-60 t^{2} G(1) .
$$

This shows that

$$
\begin{aligned}
0=H(0,0,0,1) & =\int_{0}^{1} H^{(3)} \\
& =\int_{0}^{1}-\frac{t}{3}\left(\int_{-t}^{t} F^{(4)}\right)-60 t^{2} G(1) \\
& \geqslant \int_{0}^{1}-\frac{t}{3} 2 t N-60 t^{2} G(1) \\
& =-\frac{2}{3}(N+90 G(1)) \int_{0}^{1} t^{2} \\
& =-\frac{2}{3}(N+90 G(1)) \frac{1}{3} .
\end{aligned}
$$

Hence, $N \geqslant-90 G(1)$. Similarly, $0 \leqslant-\frac{2}{9}(-N+90 G(1))$. Consequently, $90 G(1) \leqslant$ $N$. We conclude that $|90 G(1)| \leqslant N$.

A similar argument works to justify e.g. Romberg's integration method [9] which generalizes Simpson rule. 


\section{Acknowledgements}

We thank Henri Lombardi for pointing us towards the Genocchi formula and we thank the referees for suggestions that helped to improve the presentation of the article.

The research leading to these results has received funding from the European Union's 7th Framework Programme under grant agreement nr. 243847 (ForMath).

\section{References}

[1] M Ampère, Analise transcendante. Essai sur un nouveau mode d'exposition $d u$ calcul différentiel $d u$ calcul aux différences et de l'interpolation des suites, considérées comme dérivant d'une source commune, Annales de Mathematiques Pures et Appliquees 16 (1825-1826), 329-349; available at http://babel.hathitrust.org/cgi/pt?id=nyp.33433062736735;seq=347;num=329.

[2] E Bishop, D Bridges, Constructive Analysis, Grundlehren der Mathematischen Wissenschaften Vol 279, Springer-Verlag, 1985; doi:10.1007/978-3-642-61667-9.

[3] M Bridger, G Stolzenberg, Uniform calculus and the law of bounded change, Amer. Math. Monthly 106 (1999), 628-635; doi: 10.2307/2589492.

[4] T Coquand, B Spitters, A Constructive Theory of Banach algebras, Logic and Analysis 2:11(2010), 1-15; doi: 10.4115/jla.2010.2.11.

[5] J Dieudonné, Foundations of Modern Analysis, Academic Press, 1960.

[6] J D Faires, R Burden, Numerical Methods, second edition, Brooks/Cole Publishing Co., Pacific Grove, CA, 1998.

[7] A Genocchi, Sur la formule sommatoire de Maclaurin et les fonctions interpolaires, (Extrait d'une Lettre adressée à M. Hermite), C.R. Acad. Sci. Paris 86(1878), 466-469.

[8] N Rennert, A Valibouze, Calcul de résolvantes avec les modules de Cauchy, Experimental Mathematics 8 (1999), 351-366; doi: 10.1080/10586458.1999.10504624.

[9] E Süli, D F Mayers, An Introduction to Numerical Analysis, Cambridge University Press, Cambridge, 2003; doi: 10.1017/CBO9780511801181.

Thierry Coquand, Computing Science Department, Göteborg University

Bas Spitters, Institute for Computing and Information Science, Radboud University Nijmegen 